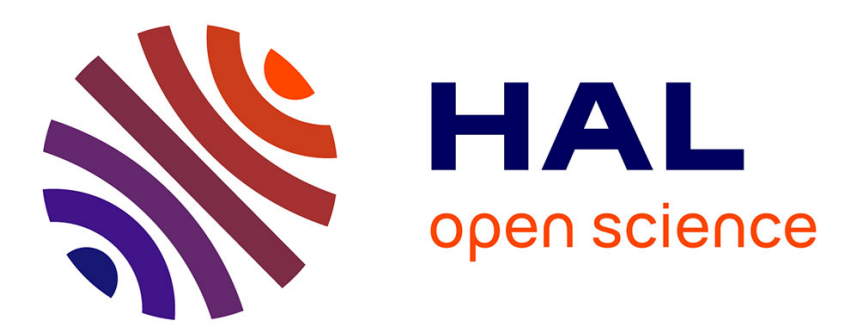

\title{
Isocrate et l'ennemi commun des Grecs : désignation et représentation des peuples d'Asie dans le corpus isocratique
}

\author{
Pascale Giovannelli-Jouanna
}

\section{- To cite this version:}

Pascale Giovannelli-Jouanna. Isocrate et l'ennemi commun des Grecs: désignation et représentation des peuples d'Asie dans le corpus isocratique. Archimède: archéologie et histoire ancienne, 2017, 4, pp.45-53. halshs-01589033

\section{HAL Id: halshs-01589033 \\ https://shs.hal.science/halshs-01589033}

Submitted on 18 Sep 2017

HAL is a multi-disciplinary open access archive for the deposit and dissemination of scientific research documents, whether they are published or not. The documents may come from teaching and research institutions in France or abroad, or from public or private research centers.
L'archive ouverte pluridisciplinaire HAL, est destinée au dépôt et à la diffusion de documents scientifiques de niveau recherche, publiés ou non, émanant des établissements d'enseignement et de recherche français ou étrangers, des laboratoires publics ou privés. 


\section{ARCHIMĖDE N ${ }^{\circ} 4$}

\section{DOSSIER THÉMATIQUE 1 : NOMMER LES « ORIENTAUX » DANS L'ANTIQUITÉ}

1 Dominique LENFANT, Agnès MOLINIER ARBO et Pascale GIOVANNELLI-JOUANNA Nommer les « Orientaux » dans I'Antiquité : présentation du dossier

6 Luca MACALE et Francesco MARI

Le lexique grec de I'Oriental dans la poésie lyrique archaïque et chez Eschyle

19 Dominique LENFANT

Les « Asiatiques » du traité hippocratique Airs, Eaux, Lieux ont-ils été les premiers « Orientaux »?

26 Yannick MULLER

Le monde « oriental » et ses habitants chez Thucydide

35 Emanuele PULVIRENTI

Des désignations des «Orientaux » chez Xénophon ? Le cas des Helléniques et de I'Anabase

45 Pascale GIOVANNELLI-JOUANNA

Isocrate et l'ennemi commun des Grecs : désignation et représentation des peuples d'Asie dans le corpus isocratique

54 Charlotte LEROUGE-COHEN

Aristote, la Politique et les « habitants de l'Asie »

60 Dominique LENFANT

A la recherche des Orientaux dans I'œuvre d'Athénée

68 Jean-Luc VIX

L'Orient chez Ælius Aristide

73 Agnès MOLINIER ARBO

Ammien Marcellin. L'Orient et les Orientaux dans l'Empire au Iv siècle

80 Agnès MOLINIER ARBO

Le vocabulaire de I'Orient et de I'Oriental dans I'Histoire Auguste. Regards d'un Romain sur l'Est de l'Empire à la fin du IV siècle

87 DOSSIER THÉMATIQUE 2 : PRYTANÉE ET REGIA

155 ACTUALITÉ DE LA RECHERCHE : DYNAMIQUES HUMAINES ANCIENNES

216 VARIA

\section{LA CHRONIQUE D'ARCHIMÈDE}

\section{(c) creative $c$ co (1) () (}




\title{
ISOCRATE ET L'ENNEMI COMMUN DES GRECS : DÉSIGNATION ET REPRÉSENTATION DES PEUPLES D’ASIE DANS LE CORPUS ISOCRATIQUE
}

\author{
Pascale GIOVANNELLI-JOUANNA \\ Maître de conférences en langue et littérature grecques \\ Université Jean Moulin-Lyon 3 \\ UMR 5189 HiSoMA \\ pascale.jouanna@univ-lyon3.fr
}

RÉSUMÉ

La pensée politique d'Isocrate, portée par ses discours, est largement tournée vers I'Asie, l'empire du Roi et les peuples qui lui sont soumis. Cette préoccupation s'inscrit notamment dans les suites de la Paix d'Antalcidas conclue en 386 av. J.-C., dont le contenu, scandaleux aux yeux de l'Athénien, a accru la mainmise perse sur le continent asiatique. L'axe essentiel qui va alors commander le programme politique défendu par Isocrate pendant une trentaine d'années, depuis le Panégyrique (380) jusqu'au Philippe (346) et au Panathénaïque (342-339), est la construction d'un panhellénisme prônant la paix et I'union entre les Grecs, étroitement subordonné à une expédition commune contre l'ennemi héréditaire perse. Comment le corpus isocratique désigne-t-il lexicalement les populations asiatiques ? De quelle vision de l'Asie et du caractère de ses habitants ces dénominations témoignent-elles?

Après avoir mis en évidence le caractère schématique et stéréotypé des modes de désignation et de

Mots-CLÉS

Antalcidas,

Asie,

Asiatiques,

barbares,

genre épidictique,

genre symbouleutique,

Grecs,

Isocrate,

lexique,

panégyrique,

panhellénisme,

Philippe,

rhétorique. représentation des peuples d'Asie dans I'œuvre d'Isocrate, l'étude montre que cette représentation ne doit pas être jugée per se comme simpliste et réductrice. Elle est en réalité à interpréter comme le support privilégié à la fois du projet politique défendu par l'Athénien et de la forme choisie par lui, à savoir une rhétorique à la croisée des genres épidictique et symbouleutique.
Isocrates' political thought, carried by his speeches, is largely turned towards Asia, the King's empire and the people who are subjects to him. This concern is part of the aftermath of the Peace of Antalcidas concluded in 386 BC., whose content, scandalous in the eyes of the Athenian, has increased the Persian seizure on the Asian continent. The essential axis which will then command the political program defended by the Athenian for some thirty years, from Panegyricus (380) to Philippus (346) and Panathenaicus (342339 ), is the elaboration of a panhellenism advocating peace and unity between the Greeks, closely subordinated to a common expedition against the Persian hereditary enemy.

How does the Isocratic corpus refer lexically to the Asian populations? What vision of Asia and the character of its inhabitants do these denominations reveal? Having shown the schematic and stereotyped nature of the modes of designation and representation of the Asian peoples in Isocrates'works, the study shows that this representation should not be judged per se as simplistic and reductive. It is in fact to be interpreted as the privileged support for both the political project he defended and the form chosen by him, namely a rhetoric at the crossroads of the epidictic and deliberative genres.

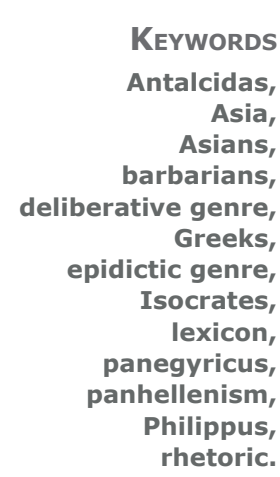


Né en 436 av. J.-C. et mort en 338 [1], au lendemain de la bataille de Chéronée, qui vit la défaite des cités grecques coalisées devant Philippe et la perte de leur indépendance, I'Athénien Isocrate a exercé durant sa longue vie trois formes d'activité au sein de sa cité [2]. Issu d'une famille aisée, mais ruinée après la défaite d'Athènes en 404, en un premier temps, il met à profit la formation rhétorique reçue de maîtres sophistes, Gorgias et Prodicos notamment [3], en s'adonnant au métier de logographe pour gagner sa vie [4]. Puis il fonde sa propre école (c. 390) en réaction contre I'enseignement des sophistes et la dirige jusqu'à la fin de sa vie. Cette création s'accompagne de la publication du manifeste Contre les sophistes (c. 391-390), et I'on peut rattacher à son travail de pédagogue la composition des deux éloges paradoxaux, Eloge d'Hélène (c. 390) et Busiris (c. 390 ou 380-378). Parallèlement à son enseignement, Isocrate veut exercer une influence sur la vie politique d'Athènes sans toutefois y participer activement : il met son activité littéraire au service des affaires de la cité, dans lesquelles il s'investit davantage en intellectuel et en conseiller politique qu'en rhéteur. De fait, dans la vie communautaire athénienne régie par l'oralité, lui qui n'a jamais prononcé ses discours, de son propre aveu à cause de sa timidité et de son manque de voix, expose ses idées dans des discours politiques écrits, depuis le Panégyrique (380) jusqu'au Philippe (346) et au Panathénaïque (342-339), ses

[1] Toutes les dates données dans le corps du texte s'entendent désormais av. J.-C.

[2] Synthèse biographique et bibliographique complète dans López Cruces \& Fuentes Gonzáles 2000.

[3] Voir par exemple Cicéron, Orator, 176.

[4] Six plaidoyers judiciaires ont été conservés, datables entre 403 et 390 : Contre Euthynous, Contre Callimachos, Contre Lochitès, Sur l'Attelage, Trapézitique, Éginétique. Isocrate niera ou passera sous silence cette activité à but alimentaire dans son discours autobiographique Sur I'Échange, 2 et 161 sqq. thèmes de prédilection se trouvant rassemblés dans son œuvre testament, Sur l'Échange (353) [5].

La pensée d'Isocrate, dépassant le cadre athénien, est largement tournée vers la politique étrangère [6]. L'axe essentiel qui la régit est la construction d'un panhellénisme prônant la paix et l'union entre les Grecs [7] à seule fin de mener la guerre contre I'ennemi commun perse. La réflexion politique de I'Athénien est par conséquent fortement tournée vers I'Asie, I'empire du Roi et les peuples qui lui sont soumis. Elle l'est d'autant plus que l'actualité contemporaine d'Isocrate a vu en 386 la conclusion du traité de paix négocié par le Spartiate Antalcidas avec le Roi Artaxerxès [8]. La Paix du Roi affirme la souveraineté perse sur les cités grecques d'Asie, et certaines îles dont Clazomène et Chypre [9]. Isocrate, dans le Panégyrique, s'insurge contre ce traité qu'il juge scandaleux en raison de I'abandon des Grecs d'Asie et de la mainmise perse accrue sur le continent asiatique [10]. Ainsi la doctrine constante d'Isocrate de guerre commune contre l'ennemi perse et le contexte international de la première moitié du IV ${ }^{\mathrm{e}}$ siècle font que I'Asie, en tant qu'espace et pouvoir, est au cœur de ses discours politiques.

Comment Isocrate désigne-t-il concrètement et verbatim les populations asiatiques ? Quelles interprétations peut-on tirer de cette approche lexicale et de quel sens est-elle porteuse ? Autrement dit, de quelle vision du monde le corpus isocratique

[5] Sur rhétorique et politique chez Isocrate, voir MASARACCHIA 1995.

[6] Sur la pensée politique d'Isocrate en matière de politique étrangère, voir MATHIEU 1966, p. 51-112.

[7] Pour une synthèse définissant le panhellénisme d'Isocrate, voir notamment BOUCHET 2014, p. 132-154.

[8] Xénophon dit en faire une citation littérale dans les Helléniques $(\mathrm{V}, 1,31)$.

[9] Contexte, contenu précis et analyse de la paix d'Antalcidas : voir par exemple CARLIER 1995, p. 31-35. [10] Panégyrique, 179. 
rend-il compte ? Enfin, quelle représentation des Asiatiques ressort de ses discours ? Ces différents niveaux permettront d'évaluer le degré de stéréotypie ou d'originalité de cet aspect de la pensée isocratique. L'analyse ne doit cependant jamais perdre de vue la part de rhétorique à l'œuvre dans l'exposé que fait l'Athénien de son programme politique. De fait, son argumentation s'appuie sur tous les effets ressortissant à la fois au genre symbouleutique (ou délibératif ) par son contenu de conseil et au genre épidictique par sa dimension d'éloge.

\section{DÉSIGNER LES PEUPLES D'ASIE : APPROCHE LEXICALE}

De quelle façon les discours isocratiques désignentils les populations orientales ?

Cette entrée en matière lexicale permet une première constatation : I'emploi du nom ßápßapos (143 occurrences) I'emporte très majoritairement sur les autres modes de désignations, différentes formes de périphrases notamment. En outre, le Panégyrique recèle à lui seul 45 , soit un tiers des occurrences [11]: de fait, dans le premier de ses grands manifestes politiques, publié en 380, une dizaine d'années après avoir commencé à y travailler (c. 392), Isocrate plaide avec force en faveur de I'union de tous les Grecs et du projet d'expédition commune en Asie contre les Perses.

\section{LES EMPLOIS DE BÁPBAPOE}

Dans la droite ligne de la représentation du monde consécutive aux guerres médiques, le corpus isocratique témoigne d'une division simplifiée de la terre habitée en deux blocs : l'Europe et I'Asie, les Grecs ("E $\mathrm{\lambda} \lambda \eta \eta \nu \varepsilon S$ ) et les barbares

[11] Contre respectivement 28 et 29 dans ses deux derniers discours, Philippe et Panathénaïque, le dernier tiers des occurrences se répartissant dans les autres discours.

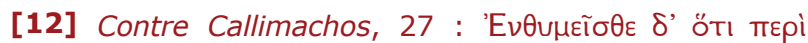

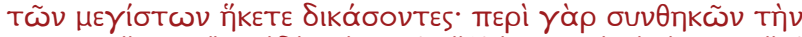

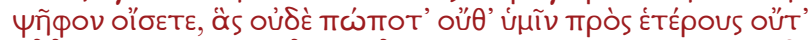

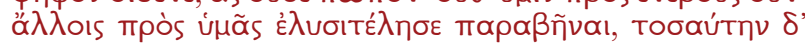

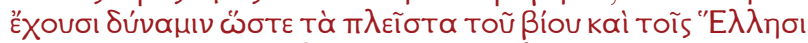

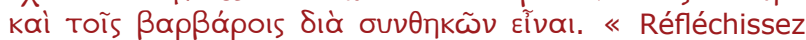
que vous êtes venus pour juger des éléments de la plus haute importance ; en effet, vous allez voter au sujet de conventions qu'il vaut mieux ne jamais transgresser, ni vous à l'égard d'autres personnes, ni autrui à votre égard, et qui ont une efficacité si grande que la plupart des affaires de la vie, pour les Grecs comme pour les barbares, se règlent au moyen de conventions. » (Traduction personnelle ici comme dans l'ensemble du document).

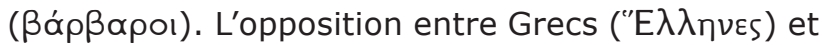
barbares ( $\beta \alpha \alpha \rho \beta \alpha \rho o$ ) est récurrente et stéréotypée dans son corpus. Le terme est rarement utilisé dans son extension maximale, quand I'addition des deux entités, Grecs et barbares, désigne le monde entier. C'est le cas dans des considérations générales, comme lorsque Isocrate, dans le plaidoyer Contre Callimachos (\$27), fait I'éloge du recours universel aux arrangements et compromis entre particuliers ou avec l'État [12], ou bien dans des sentences : «sachant que, chez les Grecs comme chez les barbares, ceux qui sont les plus renommés pour leur valeur possèdent les biens les plus

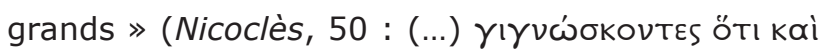

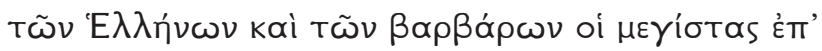

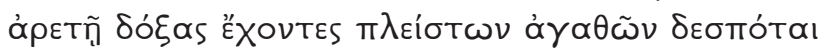

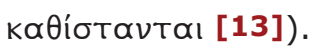

De façon générale néanmoins, l'appellation de « barbares » est employée dans une extension moindre pour renvoyer implicitement aux Perses et aux peuples de l'empire perse [14]. Deux contextes sont privilégiés : dans le passé, les guerres médiques et les victoires des Grecs sur l'ennemi perse, où, par exemple, sans être nommé,

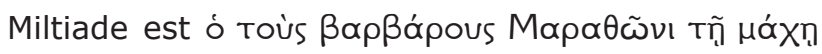
vıkńoas, « celui qui a vaincu au combat les barbares à Marathon »[15], et dans le présent, le projet d'expédition commune défendu avec constance par Isocrate, comme dans le Sur l'Échange, 77 : « En outre, quel discours (i.e. d'Isocrate) porterait sur un sujet plus beau et plus important que celui qui invite les Grecs à une expédition contre les barbares et conseille la concorde mutuelle ? » ("ETı $\delta \dot{\varepsilon ̀ ~ T i ́ s ~}$

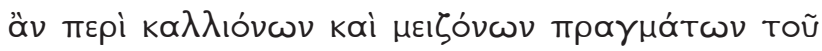
тoùs "E

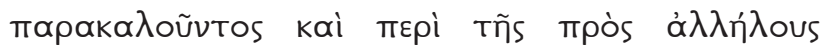

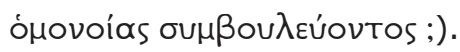

[13] Le texte grec auquel nous renvoyons est l'édition d'Isocrate dans la CUF : MATHIEU \& BRÉMOND 1929-1967.

[14] Il est toujours précisé quand le terme a son acception plus large ; ainsi, dans Panégyrique, 67, les barbares sont les Scythes, les Thraces et les Perses, autrement dit les barbares d'Europe et ceux d'Asie. Il en est de même quand il s'agit des « barbares d'Europe », comme dans Philippe, 152, s'agissant des campagnes du roi macédonien contre les Thraces, Péoniens et Illyriens (voir n. ad loc. p. 59 dans l'édition de Mathieu et Brémond pour la CUF).

[15] Sur I'Échange, 306. Voir aussi par exemple Archidamos, 43 (les Athéniens face à la force des Barbares,

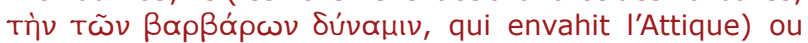
Panégyrique, 87 (le débarquement des barbares, тทेv

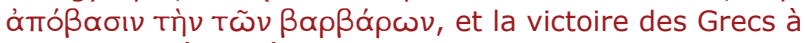
Salamine et à l'Artémision). 
Le nom est le plus souvent utilisé au pluriel

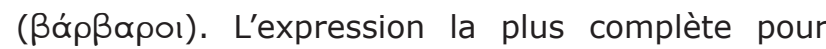
rendre la mosaïque des peuples d'Asie sujets du Roi

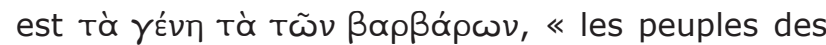
barbares » (Panathénaïque, 119).

Employé seul au singulier, ó $\beta a ́ p \beta \alpha \rho o s$ désigne soit métonymiquement le collectif des peuples d'Asie

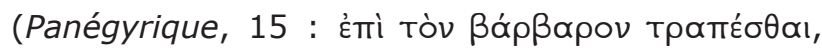
« se tourner contre le Barbare » pour évoquer l'expédition contre l'empire perse) [16], soit le Roi lui seul (Archidamos, 27 [17]).

À deux reprises seulement et dans I'unique discours

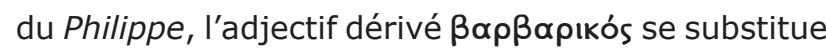

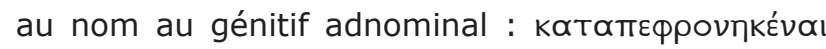

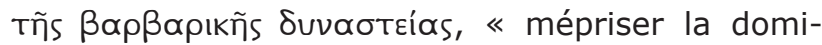

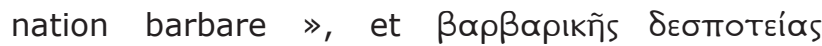

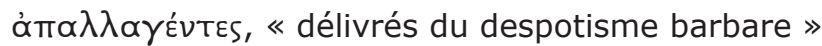
(Philippe, 100 et 154). Cette convergence n'est sans doute pas un hasard. Pour Isocrate, Philippe de Macédoine est le seul à pouvoir abattre la puissance barbare ; il lui demande donc de prendre l'initiative de monter l'expédition contre eux. La présence des deux variantes dans cette seule composition n'est pas à interpréter sur le fond : elle est clairement liée à la recherche d'effets rhétoriques dans ce discours de la maturité qu'Isocrate consacre à I'homme de la dernière chance pour être I'hêgemôn des Grecs. Dans une prose très travaillée, Isocrate martèle les moments-clés de son éloge d'allitérations (en gutturale sourde $\mathrm{k}$ ) pour emporter la persuasion : c'est le cas quand il évoque avec dédain le piètre Roi ennemi, auquel les cités grecques n'obéissent pas et dont Philippe triomphera par conséquent aisé-

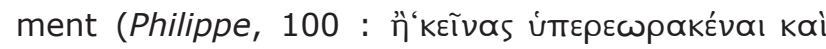

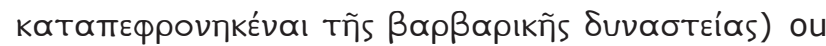
bien à la fin de l'éloge, lorsqu'il clame les bienfaits attendus de l'expédition menée par Philippe contre les Perses et la reconnaissance universelle dont le roi jouira, en scandant sa conclusion avec des parallélismes et de subtiles variations (154: $\beta \propto \sigma ı \lambda_{ı} \tilde{\omega}_{\mathrm{s}}$

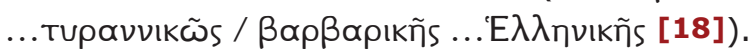

Dans le corpus isocratique, l'empire perse est lui-même assimilé à I'Asie : c'est ainsi qu'à côté d'Amyntas, roi des Macédoniens, et de Denys, tyran de Sicile, le Roi est désigné par la périphrase Tũ

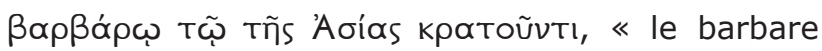
maître de I'Asie » (Panégyrique, 126 et 178) [19]. Il n'est pas anodin pour notre propos de remarquer que, s'il cite nommément ici les gouvernants de Macédoine ou de Sicile, ou ailleurs les Rois perses historiques, Darius et Xerxès, Isocrate ne cite jamais le nom d'un Roi contemporain. Cette absence participe de la schématisation avec laquelle procède Isocrate dans sa représentation des Asiatiques, ainsi que nous le verrons dans les deux dernières parties (voir infra).

Le rhéteur recourt aussi à des périphrases simples (sans $\beta \alpha ́ \rho \beta \alpha \rho o s)$ associées à l'Asie pour désigner des hommes ou plus largement des peuples du continent et de l'empire.

\section{LES PÉRIPHRASES DU TYPE

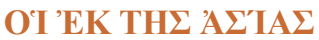

Il faut avant tout préciser que le toponyme «Asie », comme celui $\mathrm{d}^{\prime}$ « Europe » du reste, renvoie souvent à lui seul par métonymie aux peuples qui habitent et composent son territoire, c'est-à-dire aux peuples asiatiques. À titre d'exemple, dans l'éloge qu'il lui consacre, Isocrate s'emploie à glorifier le roi de Chypre Évagoras en magnifiant ses actions : au contraire des héros qui, avec l'appui de toute la Grèce, ont pris Troie, lui, qui n'avait qu'une seule cité, « a fait la guerre contre I'Asie tout entière »

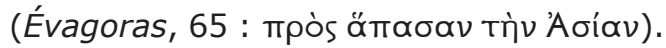

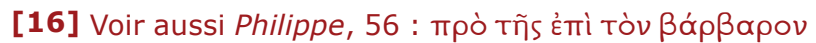
бтратвías, « avant l'expédition contre le Barbare ».

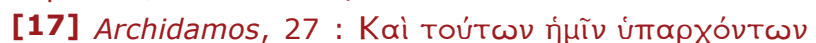

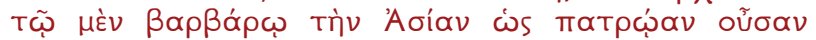

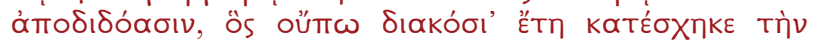
ápxìv, «Alors que nous sommes dans cette situation, ils livrent I'Asie, sous prétexte qu'elle est un héritage ancestral, au barbare qui possède le pouvoir depuis moins de deux cents ans. » (grand Roi au sens générique ici). Voir aussi Panégyrique, 163.

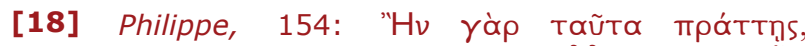

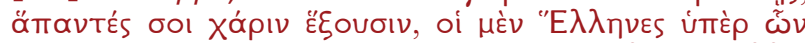

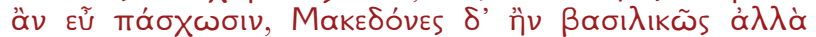

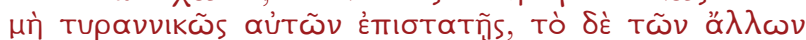

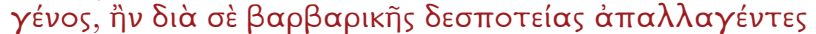

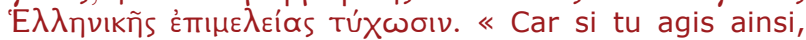
tous les hommes sans exception auront pour toi de la reconnaissance, les Grecs pour les bienfaits qu'ils en retireront, les Macédoniens si tu les gouvernes en roi et non en tyran, le peuple des autres si, délivrés par toi d'un despotisme barbare, ils obtiennent un gouvernement comme en ont les Grecs. »

[19] Panéyrique, 126 (les Lacédémoniens entre 382

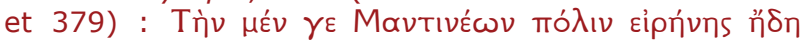

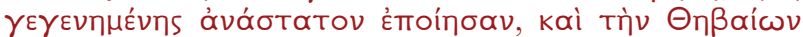

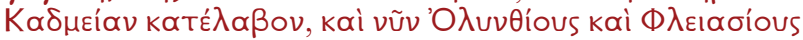

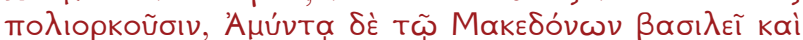

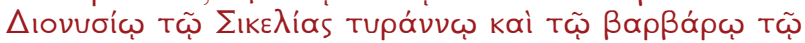

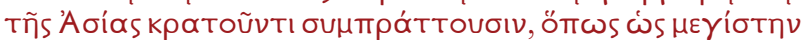

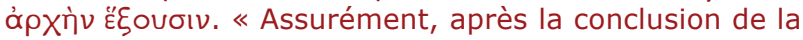
paix, ils ont mis en ruine la cité de Mantinée et se sont emparés de la Cadmée de Thèbes; et aujourd'hui, ils assiègent Olynthe et Phlionte et aident Amyntas, le roi de Macédoine, Denys, le tyran de Sicile, et le barbare maître de I'Asie, afin de posséder l'empire le plus grand possible. » 
Il est à noter ensuite qu'aucun adjectif composé du type 'Aøı le corpus isocratique. En revanche, ce dernier compte diverses périphrases (peu nombreuses au regard des occurrences de $\beta \alpha$ óp $\beta \alpha \rho \varsigma$ ) dépendant de l'article sur le schéma « article suivi d'un complément », « ceux d'Asie » désignant les sujets du Roi. Ainsi, Xerxès conduit la seconde expédition

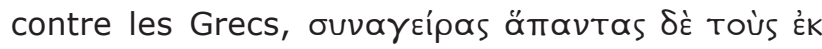
Tñs 'A oías, " après avoir rassemblé tous les peuples d'Asie sans exception » (Panégyrique, 88) [20].

Cette périphrase présente des variantes du type oi tท̀v 'A бíav katoikoũvtes, " les habitants/populations d'Asie » (participe substantivé) [21]. Ainsi, évoquant I'expédition des Dix Mille, Isocrate souligne la piètre valeur de l'armée du Roi après la mort de Cyrus à Counaxa : «Mais quand, après la mort de Cyrus, tous les peuples d'Asie sans exception eurent joint leurs forces, dans ces circonstances favorables, ils combattirent si honteusement qu'ils ne laissèrent aucun argument à ceux qui ont I'habitude de vanter le courage

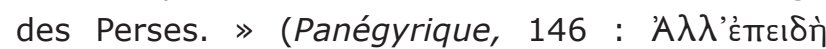

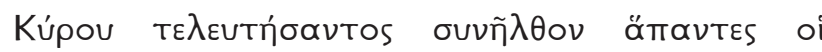

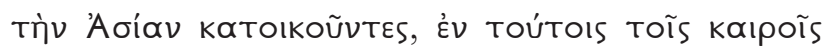

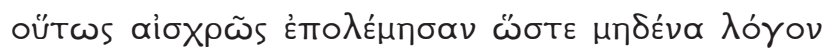

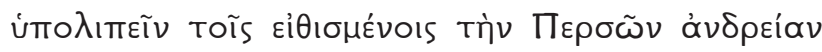

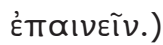

On notera la variante oi Ėmì $\theta \alpha \lambda$ átтṇ, « les peuples du bord de mer » (Panégyrique, 145) pour désigner les satrapies maritimes du parti du Roi dans l'évocation de l'expédition des Dix Mille.

Il arrive que, dans des contextes clairement identifiés, la même périphrase désigne les Grecs d'Asie, habitants de la frange maritime de I'Asie, comme c'est le cas dans l'évocation de la paix d'Antalci-

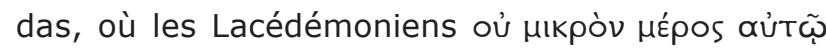

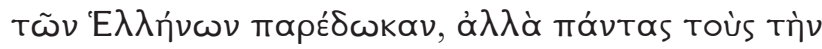
A đíav oíkoũvtas, « lui (ie au Roi) livrèrent non une petite partie des Grecs, mais tous les Grecs qui habitaient l'Asie » (Panathénaïque, 106) [22].

[20] Autre exemple dans Panégyrique, 82 (éloge des combattants des guerres médiques): Tolaútaıs

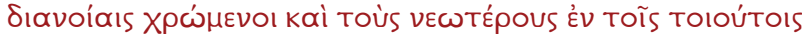

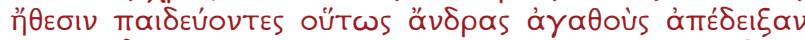

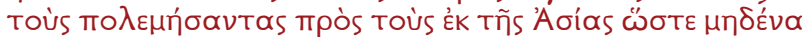

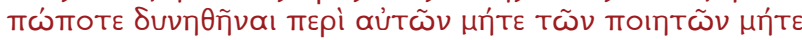

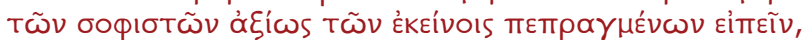
« Forts de telles conceptions et élevant les jeunes gens dans de telles habitudes, ils (i.e. les Grecs) firent de ceux qui avaient fait la guerre aux peuples d'Asie des hommes si braves qu'aucun poète ni rhéteur n'a jamais pu s'exprimer à leur sujet d'une manière qui fût à la hauteur de leurs actions. »

\section{L'ANTONOMASE

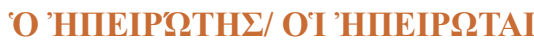

De même que le nom commun í n̋́reıpos, «le continent », est employé pour signifier I'Asie

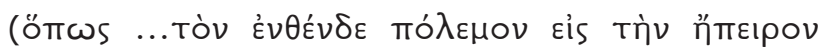

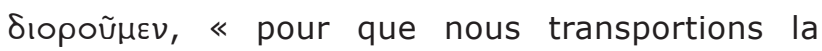
guerre d'ici sur le continent », Panégyrique, 174),

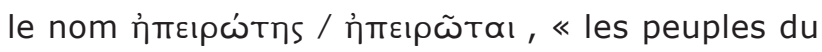
continent (litt. les continentaux) 》 désigne les Asiatiques. Ainsi, « les Perses gouvernent le conti-

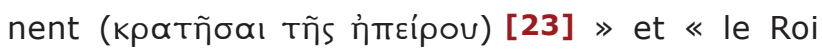
commande aux peuples du continent », $\beta \alpha \sigma ı \lambda \varepsilon \dot{s}$

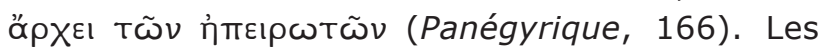
quatre occurrences du corpus se trouvent dans le Panégyrique $(132,157,166,187)$, ce qui ne surprend pas. En effet, ce discours, qui possède une forte unité [24], ne perd jamais de vue son double objet - concorde des Grecs et guerre commune contre les Perses - et consacre au second un argumentaire continu du § 129 jusqu'à la fin du discours au $§ 189$.

\section{L'ETHNONYME II'EPEAI}

Le seul ethnonyme présent dans le corpus isocratique pour désigner les Asiatiques est Пépoa। (12 occurrences), ainsi que l'adjectif dérivé Пєрбıкós (10 occurrences) ; ils désignent en général par métonymie l'ensemble des peuples asiatiques constituant I'empire, et non I'ethnie perse en particulier [25].

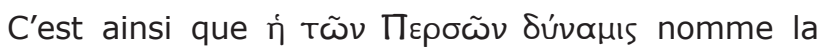
puissance de I'Asie du Roi (Nicoclès, 23). La même remarque peut être faite à propos de l'adjectif dans

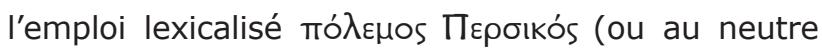
pluriel) [26] pour signifier les guerres médiques.

Cette première étape fondée sur l'examen du lexique montre qu'Isocrate recourt très majoritairement au terme «barbares», avec sa variante « barbares d'Asie », pour désigner les peuples habitant I'Asie. En comparaison, les périphrases plus précises ou les ethnonymes sont rares. La dénomination des populations asiatiques apparaît

[21] Voir aussi Panégyrique, 83 et 146; Panathénaïque, 83. [22] Idem dans le même contexte du traité de 386 : Lettre à Archidamos, 8 : oi tñs Aoías triv mapa入íav oíkoũvtes, « les habitants de la côte de l'Asie » (Grecs).

[23] Archidamos, 26.

[24] Sur la composition du discours, voir MATHIEU 20037, p. 8-9.

[25] Dans le contexte particulier de la constitution de l'empire perse, Cyrus et les troupes perses soumettent

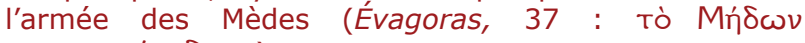

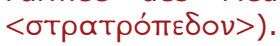

[26] Voir par exemple, Panégyrique, 68 ; Paix, 37 et 88. 
par conséquent comme schématique et stéréotypée [27]. Cependant, cette approche lexicale a déjà permis d'observer qu'il ne faut pas négliger les aspects rhétoriques présidant au recours à tel ou tel mot, comme l'a montré l'analyse de la récurrence

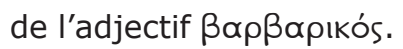

\section{L'ASIE ET SES PEUPLES, PARTIE D'UN MONDE BIPOLAIRE ET ANTAGONISTIQUE}

Le corpus isocratique témoigne d'une représentation essentiellement binaire et bipolaire du monde. Ce dernier est composé de deux blocs monolithiques, I'Europe et I'Asie, respectivement assimilées aux Grecs et aux Barbares [28], I'unité de chacune étant souvent renforcée et rhétoriquement appuyée par l'adjectif örmas [29] : « En effet, tandis que toute la terre située sous le ciel est divisée en deux parties, I'une appelée I'Asie, l'autre I'Europe, <le barbare> en a reçu la moitié par le traité (...). »

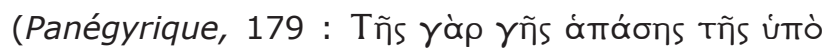

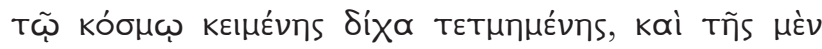

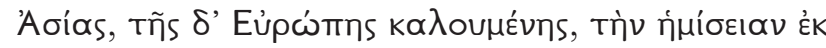

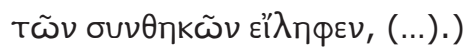

De fait, I'intérêt de l'Athénien est peu porté vers les autres contrées [30], en particulier vers l'Ouest, sans doute ressenti comme lointain et éloigné de la cause panhellénique [31]. Cette lecture bipolaire du monde est donc le support privilégié de la cause panhellénique défendue par Isocrate, qui promeut aussi I'union et la paix entre Grecs pour revenir sur toute autre répartition des forces (certains Grecs, dont les Spartiates, alliés aux Barbares).

Affirmant un point de vue athénocentré [32], Isocrate est donc le partisan d'un impérialisme athénien orienté vers I'Est et I'Asie. Les deux blocs sont antagonistes et luttent chacun pour l'hégémonie. Isocrate pense ainsi leurs rapports en termes de domination et de soumission, d'esclavage et de servitude. Témoin, par exemple, I'expression de son exaspération au sujet de la paix de 386 qui a accentué cette bipartition en renforçant, du point de vue athénien en tout cas, le poids du bloc asiatique :

«N'allons-nous pas vers lui comme vers un maître pour nous accuser mutuellement ? Ne l'appelons-nous pas le grand Roi comme si nous étions devenus ses captifs ? » (Panégyrique, 121 : Oủx

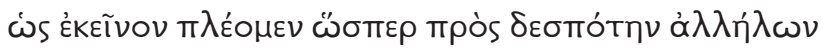

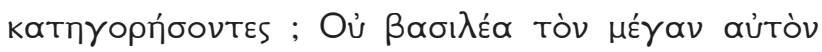

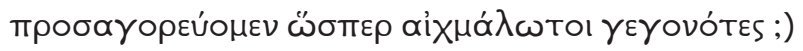

La rhétorique isocratique sait user de tous ses effets pour évoquer la guerre commune qu'avec les Lacédémoniens il faudrait mener contre le Roi et ses peuples sujets. Adaptant remarquablement son propos à son objet, Isocrate recourt à l'expression métaphorique pour désigner la soumission des Barbares : les Lacédémoniens ont forcé

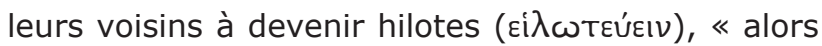
qu'ils pourraient, après s'être réconciliés avec nous, faire de tous les Barbares les périèques de la Grèce

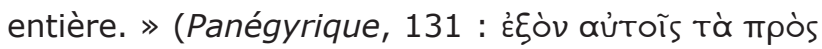

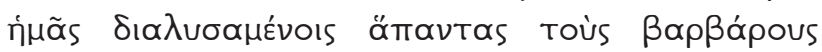

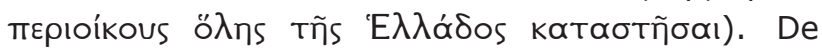
même, plus tard, Philippe devra contraindre « les

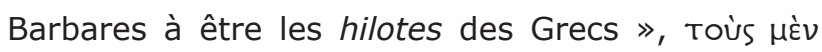

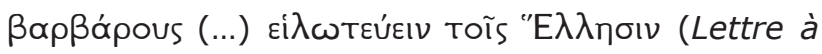
Philippe III, 5). Le recours à I'image des périèques et des hilotes dit avec force et expressivité la soumission des Barbares par les Grecs.

Dans les discours d'Isocrate, la représentation bipolaire du monde opposant Grecs et Barbares d'Asie, largement héritée des guerres médiques, est rétroprojetée sur la période mythique. Les temps mythiques sont revisités et réinterprétés à I'aune des guerres contre les Perses : c'est ainsi que l'Éloge d'Hélène (\$51) présente la guerre de Troie comme un conflit entre I'Europe et I'Asie, et non entre Achéens et Troyens [33], ou entre Ménélas et Alexandre :

« Nous pouvons légitimement penser que nous devons aussi à Hélène de ne pas être les esclaves des Barbares. De fait, nous reconnaîtrons que, grâce à elle, les Grecs se sont unis et ont organisé
[27] Participe aussi de cette schématisation, comme nous l'avons vu supra, I'anonymat constant du Roi, qui à l'exception des trois Rois historiques Cyrus, Darius et Xerxès, n'est jamais nommé autrement que par sa fonction. [28] Cf. Panégyrique, 179: le Roi a reçu la moitié du monde.

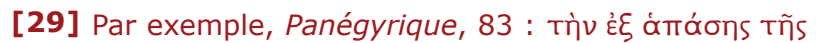

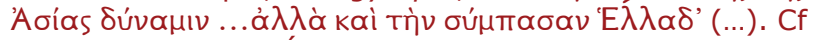
aussi Nicoclès, 65, Évagoras, 71, etc.

[30] Par exemple, la Libye et l'Égypte dans le Busiris ou le Nord et la Thrace dans la Paix. Voir BOuCHET 2014, p. $146-9$ et $150-1$.

[31] Voir BOUCHet 2014, p. 149-150.

[32] Sur ce point, qui n'est pas une originalité d'Isocrate, cf. le corpus des orateurs attiques, dont les aspects idéologiques de propagande athénienne ont été particulièrement étudiés dans LORAUX 19932. Sur I'athénocentrisme d'Isocrate, voir BOUCHET 2014, p. 152-153.

[33] D'où I'analogie entre Perses et Troyens : sur la question, voir LENFANT 2004. 
une expédition commune contre les Barbares, et qu'alors pour la première fois, I'Europe a dressé un

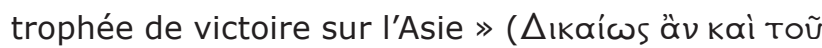

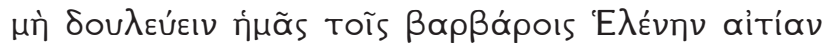

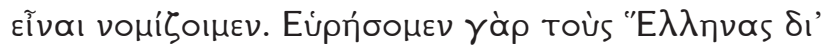

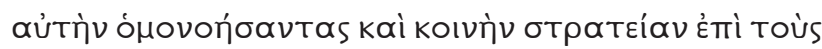

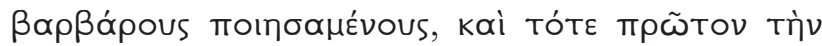

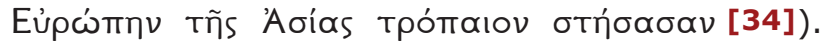
Initialement dirigé contre Troie, le conflit est dans ce schéma réinterprété comme une guerre des Grecs contre toute I'Asie. De la même manière, I'expédition d'Héraclès contre Troie [35] est menée contre la cité forte de «la plus grande puissance

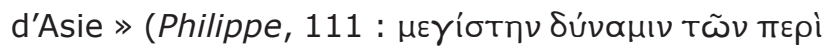
тท̀v Aoíav).

En fait, cette lecture rétrospective des guerres mythiques, qui actualise les Troyens en Barbares d'Asie, pour stéréotypée qu'elle soit, participe de I'argumentation d'Isocrate en faveur d'une expédition commune contre le bloc asiatique. Les discours développent en effet deux arguments essentiels pour justifier ce projet.

Le premier, de nature rhétorique et idéologique, est que les peuples d'Asie constituent I'ennemi naturel et héréditaire des Grecs : $\mu \varepsilon \tau \grave{\alpha} \tau \tilde{\omega} \nu$

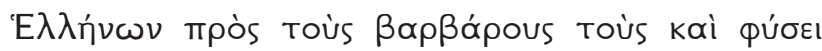

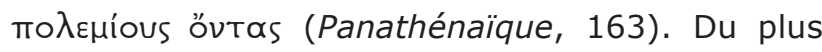
loin que I'on remonte - jusqu'aux temps mythiques précisément -, les deux blocs ont entretenu des rapports de haine mutuelle. Les Grecs vouent aux Barbares et à leurs Rois une « haine ancestrale »

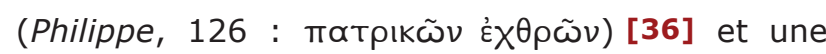
« hostilité naturelle » (Panégyrique, 158 : фúఠєl

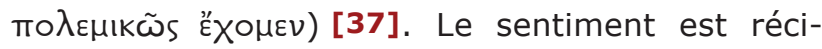
proque puisque les Barbares vouent la même haine

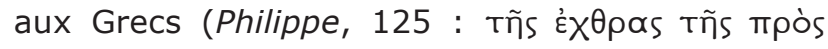
Toùs "E $\mathrm{\lambda} \lambda \eta \eta$ uas). L'argument du Barbare comme ennemi naturel et des deux blocs antagonistes est un argument rhétorique topique dans le corpus isocratique par sa récurrence. Il est simplificateur, même s'il arrive à Isocrate de nuancer et de montrer la porosité des deux parties, en évoquant par exemple les Grecs partis vivre en terre d'Asie pour y trouver leur subsistance, les mercenaires notamment (Sur la Paix, 42).

Le second argument développé par Isocrate pour justifier l'expédition commune est de nature économique. De fait, son programme politique, qui a été constant [38], comprend un plan de colonisation dont le but est d'exploiter les richesses du continent asiatique [39]. Ce projet d'annexion et d'exploitation doit aboutir à donner de nouvelles frontières à la Grèce [40], mais il est remarquable que, du Panégyrique (380) au Philippe (346), celles-ci évoluent, comme évolue aussi I'ambition du programme. Dans le Panégyrique, il s'agit de soumettre successivement chacun des peuples d'Asie (§164) pour exploiter à terme l'Asie tout

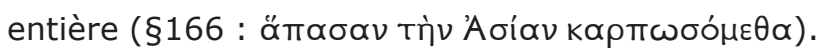
Quelque trente ans plus tard, s'il semble toujours en apparence prôner l'anéantissement complet de la royauté perse, le Philippe envisage à défaut (§120: عỉ $\delta \dot{\varepsilon} \mu \eta ́)$ un programme de colonisation délimité dans l'espace, mais détaillé dans son contenu. Il stipule que les Grecs coupent le territoire de I'Asie en deux, sur une ligne allant de la Cilicie à Sinope, pour y fonder des cités et y établir la population misérable et dangereuse des mercenaires ( $\$ 120$ et 122 ). La frange maritime des cités grecques d'Asie se trouverait donc libérée et la partie asiatique colonisée augmentée.

En matière de politique étrangère, Isocrate développe donc une vision bipartite et antagonistique du monde dans laquelle s'opposent les deux blocs de l'Europe, centrée sur les Grecs et Athènes, et de I'Asie. Les peuples d'Asie ne sont guère mentionnés pour eux-mêmes, mais forment une entité politique représentant l'ennemi naturel et historique des Grecs. Cette vision schématique, qui sert la rhétorique isocratique autant qu'elle en est le produit, n'est pas contredite par le portrait stéréotypé qui est fait des Asiatiques.

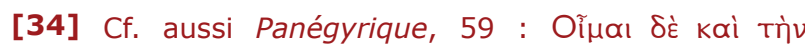

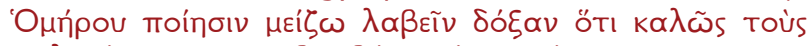

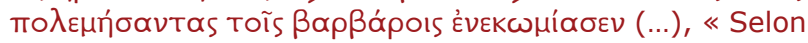
moi, la poésie d'Homère a remporté plus de gloire parce qu'il a fait un bel éloge de ceux qui ont combattu les Barbares. »

[35] Sur l'épisode mythique de la « première guerre de Troie », où Héraclès vient se venger du roi Laomédon pour n'avoir pas obtenu la récompense promise pour avoir sauvé Hésionè, voir Diodore de Sicile, Bibliothèque historique, IV, 42, 3-7 et 49, 3 ; Apollodore, Bibliothèque, II, 5, 9 et 6,4 .

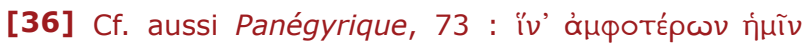

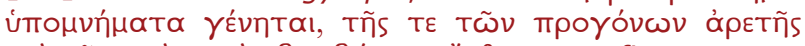

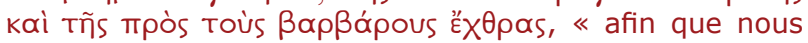
nous souvenions de deux choses, de la vaillance de nos ancêtres et de leur haine contre les Barbares. »

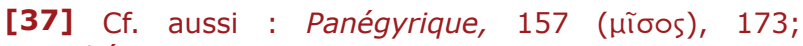
Panathénaïque, 102 etc.

[38] Voir Philippe, 130.

[39] Le verbe employé de manière privilégiée est

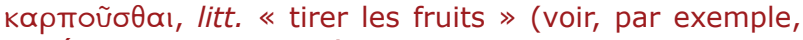
Panégyrique, $133 ; 166)$

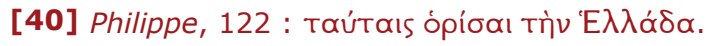




\section{UNE REPRÉSENTATION STÉRÉOTYPÉE DES ASIATIQUES}

Le portrait que dresse Isocrate des populations d'Asie dans ses discours reprend l'intégralité des stéréotypes ethniques des Barbares de ce continent, des Perses en particulier [41]. De façon générale, leurs caractéristiques d'ethos, émanant d'un point de vue hellénocentré, sont l'envers des qualités prônées par la morale grecque commune. L'image des peuples asiatiques en général, que I'Athénien représente sans la distinguer de celle des Perses stricto sensu, est veule et méprisable. La question ayant déjà été largement traitée [42], nous nous en tiendrons ici à un exposé succinct des traits essentiels. Les Barbares d'Asie sont dépourvus de courage

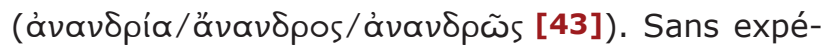

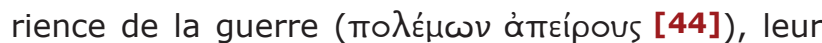
puissance au combat leur vient de leur nombre hors

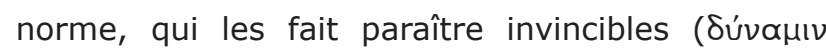
ávumóбтaтov, « force impossible à soutenir » [45]). Leur nature est servile et soumise au despotisme. Ils sont mous ( $\mu \alpha \lambda \alpha$ koús) et corrompus par le luxe

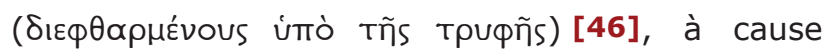
de leur richesse et de leur prospérité [47]. Leur

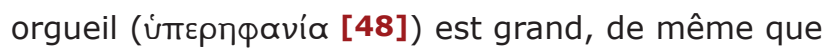

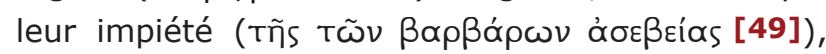
comme l'a bien montré Xerxès.

Il est à noter, comme précédemment, que la rhétorique parfois épouse le cliché et que le mépris s'exprime, non plus avec des traits stéréotypés, mais au moyen d'une expression stylistique particulière. Ainsi, à la fin du Philippe (§139), voulant avec force persuader les Grecs et le roi de Macédoine que ce dernier doit être leur hêgemôn, Isocrate évoque avec dédain le fondateur de l'empire perse, un Cyrus qu'à dessein il ne nomme pas mais rejette dans

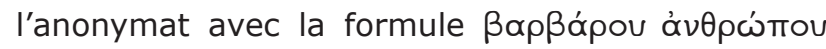

[41] Sur les Barbares dans la pensée grecque, voir Romilly 1993. Chez Isocrate spécifiquement, voir entre autres, LeNFANT 2011, p. 227-232 et BOUCHET 2014, p. 178-193.

[42] Voir note précédente pour des éléments bibliographiques non exhaustifs.

[43] Voir par exemple, Philippe, 137 et 100 ; Evagoras, 66.

[44] Philippe, 124.

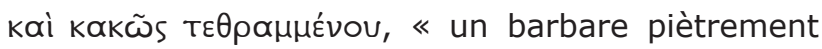
éduqué».

Par conséquent, le corpus isocratique déploie tous les clichés attachés aux Perses, c'est-à-dire aux Barbares d'Asie. Il faut bien voir cependant que ce portrait caricatural, s'il est un lieu commun de la rhétorique, est aussi là pour servir le programme panhellénique $d^{\prime}$ Isocrate [50] associé à son projet d'expédition commune. Plus la présentation de I'ennemi d'Asie est défavorable et plus l'ennemi est facile à combattre, alors plus les Grecs seront motivés pour se liguer contre lui.

\section{CONCLUSION}

L'étude des modes de désignation et de représentation des peuples d'Asie dans I'œuvre d'Isocrate a permis de mettre en évidence leur caractère schématique et stéréotypé, ainsi que leur stabilité dans l'expression de l'auteur. D'une part en effet, l'Athénien a très majoritairement recours à la dénomination de «Barbares», les autres appellations étant marginales ; d'autre part, sa conception du monde repose sur un schéma constant opposant frontalement deux blocs politiques, l'Europe, centrée sur la Grèce et Athènes, et l'Asie, équivalent à l'empire du Roi avec ses peuples sujets. Pour autant, cette représentation ne doit pas être jugée per se comme simpliste et réductrice. Elle est en réalité à interpréter comme le support privilégié à la fois de la cause défendue par Isocrate -l'expédition commune contre I'Asie adossée à une union panhellénique, soit un pôle du monde contre l'autre-, et de la forme choisie par lui, à savoir une rhétorique à la croisée de l'épidictique et du délibératif, usant de tous les effets grossissants pour persuader les Athéniens.

[45] Archidamos, 43. Cf. I'épisode des Thermopyles où les 1000 Grecs trouvèrent en face d'eux 700000 Barbares (Archidamos, 99).

[46] Philippe, 124.

[47] Philippe, 130.

[48] Voir Panégyrique, 89 et Éloge d'Hélène, 49 (oi

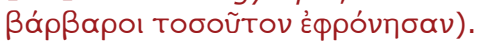

[49] Panégyrique, 156.

[50] Voir BOUCHET 2014, p. 193. 
Bouchet, Christian, 2014, Isocrate l'Athénien ou la belle hégémonie. Étude des relations internationales au IVe siècle a.C., Bordeaux.

CARLIER, Pierre, 1995, Le ive siècle grec jusqu'à la mort d'Alexandre, Paris.

Lenfant, Dominique, 2004, «L'amalgame entre les Perses et les Troyens chez les Grecs de l'époque classique : usages politiques et discours historiques », dans José Maria Candau Morón et alii (éd.), Historia e mito. El pasado legendario como fuente de autoridad, Malaga, p. 77-96.

Lenfant, Dominique, 2011, «Isocrate d'Athènes », dans Dominique Lenfant (éd.), Les Perses vus par les Grecs. Lire les sources classiques sur l'empire achéménide, Paris, p. 227-232.

López Cruces, Juan Luis \& Fuentes González, Pedro Pablo, 2000, « Isocrate d'Athènes », in Richard Goulet (éd.), Dictionnaire des philosophes antiques, t. 3, Paris, p. 891-938.

LorauX, Nicole, 1993, L'invention d'Athènes, Paris.

Masaracchia, Agostino, 1995, Isocrate. Retorica e politica, Roma.

Mathieu, Georges, 1966, Les idées politiques d'Isocrate, Paris.

Mathieu, Georges \& Brémond, Émile (éd.), 1927-1967, Isocrate. Discours, 4 vol., Paris.

Romilly, Jacqueline (de), 1993, « Les barbares dans la pensée de la Grèce classique », Phœnix 47, p. $283-292$. 